\title{
ADOLESCENTES Y TELÉFONO MÓVIL: PERSPECTIVA DE PADRES RESIDENTES EN EL ÁMBITO RURAL
}

\author{
Alba Castro Arroyo \\ Educadora Social \\ albacastro@usal.es \\ Ana Isabel Isidro de Pedro \\ Profesora Titular de E.U. Departamento de Psicología Social y Antropología \\ Universidad de Salamanca \\ anyis@usal.es
}

Fecha de Recepción: 23 Abril 2019

Fecha de Admisión: 30 Abril 2019

\section{RESUMEN}

Resulta innegable el continuo desarrollo y la abrumadora popularidad de las nuevas tecnologías de la información y la comunicación. Concretamente, el teléfono móvil se ha adentrado en nuestra cotidianeidad, convirtiéndose en un elemento imprescindible para buena parte de la población -y especialmente para los adolescentes-, adquiriendo funciones relacionales y comunicativas tan potentes que han conllevado cambios en el comportamiento psicosocial. Además, estudios al respecto avalan que esto es especialmente significativo en menores residentes en zonas rurales, dadas las especiales características del entorno (despoblación -principalmente en lo que a la población joven respecta-, déficit de infraestructuras, escasa oferta de actividades de ocio, lejanía de otros núcleos de población...) Dada esta inminente realidad, de innegables implicaciones educativas, el presente trabajo pretende analizar si los padres, madres y/o tutores residentes en el ámbito rural aprecian cambios en el comportamiento psicosocial de sus hijos -en edad adolescente y con teléfono móvil propio- debidos al uso del mismo. Para realizar la investigación se ha seleccionado una pequeña localidad rural de la comarca de La Armuña (Salamanca) y, concretamente, una muestra formada por 37 padres, madres y/o tutores, con hijos en edad adolescente, de los que el $54 \%$ son mujeres y el $46 \%$ hombres y con edades comprendidas entre los 35 y los 65 años. Por su parte, el instrumento medida utilizado es un cuestionario ad hoc, de elaboración propia, constituido por un total de 41 ítems. Los resultados revelan que, debido al uso del móvil, los padres aprecian cambios no sólo en el comportamiento de sus hijos adolescentes, sino también en la propia forma de vida y en las rutinas familiares. Igualmente, se comprueba cómo, en la muestra estudiada, pocos padres 


\section{ADOLESCENTES Y TELÉFONO MÓVIL: PERSPECTIVA DE PADRES RESIDENTES EN EL ÁMBITO RURAL}

establecen reglas o límites de uso de los dispositivos móviles a sus hijos, así como la escasa formación de los padres en las nuevas tecnologías.

Palabras clave: teléfono móvil; adolescencia; comportamiento psicosocial; ámbito rural

\section{ABSTRACT}

Teenagers and mobile telephone: the perspective of parents residents in the rural area. Teenagers and mobile telephone: the perspective of parents residents in the rural area. The continued development and overwhelming popularity of the new information and communication technologies is undeniable. Specifically, the mobile phone has entered into our daily lives, becoming an essential element for a large part of the population -and especially for adolescents-, acquiring relational and communicative functions so powerful that they have entailed changes in psychosocial behavior. In addition, studies in that regard support that this is especially significant in minors living in rural areas, because the special characteristics of the environment (depopulation -primarily talking about the young population-, infrastructure deficit, scarce offer of free time activities, distance of other population centers...) Given this imminent reality, of undeniable educational implications, this work aims to analyze whether parents, mothers and/or tutors living in rural areas appreciate changes in the psychosocial behavior of their children -in adolescent age and owning a mobile phone- due to the use of that. To carry out the research, a small rural village in the region of La Armuña (Salamanca) has been selected. The sample consisting of 37 parents, mothers and/or tutors, with children in adolescent age, $54 \%$ of which are women and $46 \%$ are men, aged between 35 and 65 years. The instrument used is an ad hoc questionnaire, prepared by the authors, consisting of a total of 41 items. The results reveal that, due to the use of the mobile phone, parents appreciate changes not only in the behavior of their adolescent children, but also in their own way of life and family routines. Likewise, it is verified that, in the studied sample, few parents establish rules or limits of using the mobile devices to their children, as well as the scarce formation of parents in the new technologies.

Keywords: mobile phone; adolescence; psychosocial behaviour; rural area

\section{ANTECEDENTES}

Resulta una realidad innegable que las nuevas tecnologías ocupan un espacio fundamental en nuestras vidas, ya que han alcanzado enormes cifras de crecimiento y popularización. Su uso nos ha facilitado realizar tareas cotidianas a las que antes dedicábamos un mayor tiempo y esfuerzo; es decir, nos simplifican los quehaceres diarios (Echeburúa y de Corral, 2010). Como resaltan Weezel y Benavides (2009), esto se debe a que se han vuelto más útiles, más efectivas y, por ello, han tenido una enorme repercusión en la sociedad de manera universalizada. Pero, sin duda, el sector más influenciado por el uso de las mismas es el de los adolescentes y el de los adultos jóvenes, según defiende Lorente (2002), el cual considera esta influencia algo más que una simple moda.

El teléfono móvil ha influido en muchos ámbitos de la sociedad. Y uno de los cambios más evidentes atañe al ámbito familiar, debido a que la introducción de estos dispositivos en su seno ha modificado las relaciones estrechas y los espacios de convivencia. A lo cual hay que añadir los importantes cambios acontecidos en los modelos familiares. En el pasado solo se permitía el modelo tradicional, pero éste cedió paso a nuevos modelos familiares muy variados y heterogéneos, que han modificado la esfera familiar y, por lo tanto, las relaciones que dentro de la misma se establecen. Uno de los avances que ha favorecido ese cambio ha sido la transformación del papel de la mujer (Valdivia, 2008). El resultado de todas estas conversiones ha sido un vacío dentro del entorno familiar, vacío que los adolescentes intentan llenar buscando otras alternativas. Aquí es donde radica la fijación, no pocas veces obsesiva, de niños y adolescentes por las nuevas tecnologías, ya 
que estas herramientas les satisfacen instantáneamente necesidades que son propias de este rango de edad. Y en ámbitos rurales ese uso telefónico destinado a llenar ese vacío se ve intensificado debido a la imposibilidad de satisfacerlo con otras actividades (Villanueva, 2012). Esto se debe a las actuales características que predominan en muchos ámbitos rurales: escasez de población menor de edad, distancia y dificultad de acceso a los núcleos urbanos, inexistencia de espacios públicos de ocio y tiempo libre, escasez o inexistencia de actividades lúdicas, etc. De tal forma que emplear el teléfono móvil como compensador de esas carencias del entorno y como sustitutivo de otras alternativas no disponibles resulta muy sencillo, práctico y reconfortante.

Obviamente, no todo el comportamiento hacia los dispositivos móviles es disfuncional; es más, las nuevas tecnologías pueden ser de gran ayuda y utilidad en nuestra vida cotidiana, haciendo un buen uso de ellas; por ejemplo, guiarnos hasta o desde una dirección, mantenernos informados de la actualidad, recordarnos nuestros eventos... Pero el uso empieza a ser negativo cuando el sujeto deja de lado sus quehaceres diarios (Echeburúa y de Corral, 2010), como estudiar, trabajar, salir con los amigos, hacer deporte, estar con la familia... Este uso negativo es más probable que sea realizado por los adolescentes que por los adultos porque entre ambos grupos existen marcadas diferencias de uso en telefonía móvil. Uno de los principales factores que marcan esta diferencia en el uso es la llamada "brecha digital generacional", que hace que adultos y adolescentes, aunque utilicen la misma función 0 aplicación del móvil, lo hagan de manera diferente y diferencial. En los adultos predomina un uso dirigido, prioritariamente, hacia cuestiones laborales y necesidades personales y menos orientado a fines sociales y, si realizan un uso social, suele ser de manera puntual (Castells y de Bofarull, 2002). Mientras que en los adolescentes predomina el uso social de las nuevas tecnologías; de hecho, se puede decir que es su objetivo principal ya que, como aseveran Castells y de Bofarull (2002, p. 145), "los adolescentes se expresan con el móvil y a través del móvil".

Por tanto, siguiendo a Cuesta (2012), reiteramos que los adolescentes son el grupo de edad que más peligro tiene respecto a la adicción y, por lo tanto, al cambio en su comportamiento debido a las nuevas tecnologías. En este sentido, una característica propia de este rango de edad es la búsqueda de experiencias nuevas y los teléfonos móviles son herramientas que pueden brindar esas sensaciones excitantes que ellos nunca habían experimentado, ya que, las emociones durante en esta etapa se encuentran a flor de piel (Graner, Beranuy, Sanchéz, Chamarro y Castellana, 2007). Y puede ocurrir que tengan escaso control cognitivo de decisión: no saben si realmente su comportamiento es bueno o malo, simplemente se centran en la gratificación inmediata que les proporciona el uso del móvil, incapaces de pensar en las consecuencias negativas que puede acarrear como, por ejemplo, el aislamiento, el desinterés por las relaciones sociales, la pérdida de tiempo, el bajo rendimiento académico o laboral, etc.

Así, corresponde a las personas más cercanas a su entorno -por regla general los padres- prestar atención a sí existen o no cambios en el comportamiento de sus hijos y establecer, desde que éstos son pequeños, pautas para realizar un uso responsable y no problemático del teléfono móvil. De esta forma, en este trabajo ponemos la mirada en padres residentes en ámbito rural para comprobar la existencia, o no, de cambios en el comportamiento psicosocial de sus hijos e hijas ligados al uso del móvil.

\section{OBJETIVOS}

\section{Objetivo general}

Comprobar si los padres, madres y/o tutores residentes en el ámbito rural aprecian cambios en el comportamiento psicosocial de sus hijos -en edad adolescente y con teléfono móvil propio- debido al uso del mismo. 


\section{ADOLESCENTES Y TELÉFONO MÓVIL: PERSPECTIVA DE PADRES RESIDENTES EN EL ÁMBITO RURAL}

\section{Objetivos específicos}

- Identificar si las rutinas familiares que antes seguían se han visto influidas o modificadas por el teléfono móvil.

- Comprobar si los menores en el ámbito rural han variado la manera de ocupar su ocio y tiempo libre, sus hobbies y sus quehaceres a raíz del uso del móvil.

- Determinar si la calidad del ocio y tiempo libre familiar ha cambiado debido al móvil.

- Identificar la utilización de reglas o límites por parte de los padres respecto al uso del móvil.

- Determinar en los padres la existencia de conocimientos o pautas funcionales para un buen uso del teléfono móvil.

\section{METODOLOGÍA}

La presente investigación se llevó a efecto a través de un cuestionario ad hoc, de elaboración propia, constituido por un total de 41 ítems, que fue aplicado a una muestra de 37 sujetos -todos ellos padres, madres y/o tutores de hijos en edad adolescente residentes en ámbito rural, concretamente en una pequeña localidad de la comarca salmantina de La Armuña- que completaron el cuestionario de manera presencial.

Una vez rellenados todos los cuestionarios, se utilizó el programa de análisis estadístico SPSS, mediante el cual se trataron y analizaron los datos.

\section{RESULTADOS Y DISCUSIÓN}

En primer lugar, respecto a las preguntas generales, como podemos observar en la Figura 1, el $35,1 \%$ de la muestra está "de acuerdo" en que su hijo tenga un teléfono móvil propio. Resalta el hecho de que a un $37,8 \%$ (suma de totalmente en desacuerdo y desacuerdo) no le gusta que su hijo tenga un móvil propio, pero aun así lo tienen.

Figura 1: Porcentajes sobre que los hijos tengan móviles propios

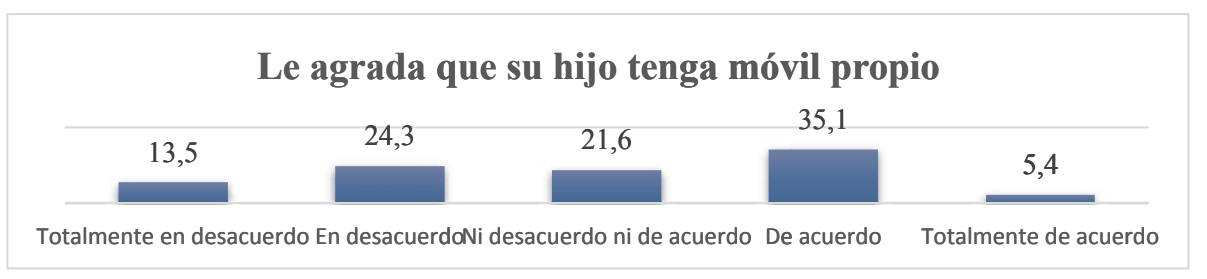

En las Figuras 2 y 3 advertimos también cómo los mayores porcentajes se corresponden a "de acuerdo" (40,5\%) y "totalmente de acuerdo" (27\%). Considerando que sus descendientes hacen un uso excesivo del móvil y, además, preocupados porque ese uso sea negativo. Como vemos es una opinión mayoritaria, ya que los menores porcentajes están en "desacuerdo" o "completamente en desacuerdo" con esta cuestión. Llama la atención el hecho de que cierto porcentaje de la muestra no se significa respecto al uso que hace su hijo con el teléfono. 
Figura 2: Porcentajes sobre el grado de acuerdo o desacuerdo sobre el exceso uso del móvil

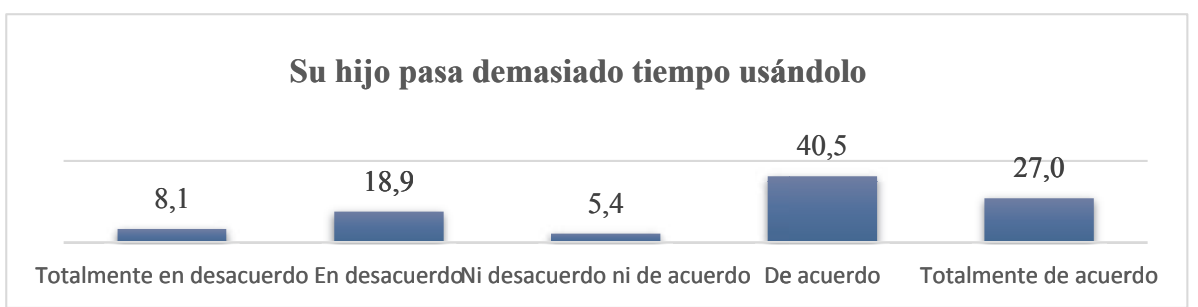

Figura 3: Porcentajes sobre la preocupación acerca del uso del móvil

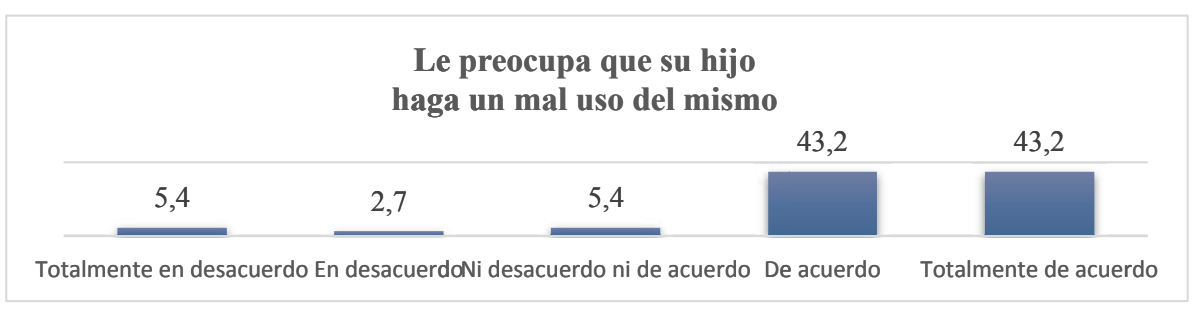

En cuanto a los sentimientos que experimentan los progenitores respecto al uso continuado que hacen sus descendientes del móvil (ver Figura 4), un 40,5\% está totalmente "de acuerdo" en que, cuando ello ocurre, sus sentimientos son de enfado. Por lo que se deduce que, en la muestra, es mayoritario experimentar sentimientos negativos cuando los descendientes hacen un uso excesivo del dispositivo.

Figura 4: Porcentajes sobre el sentimiento de enfado

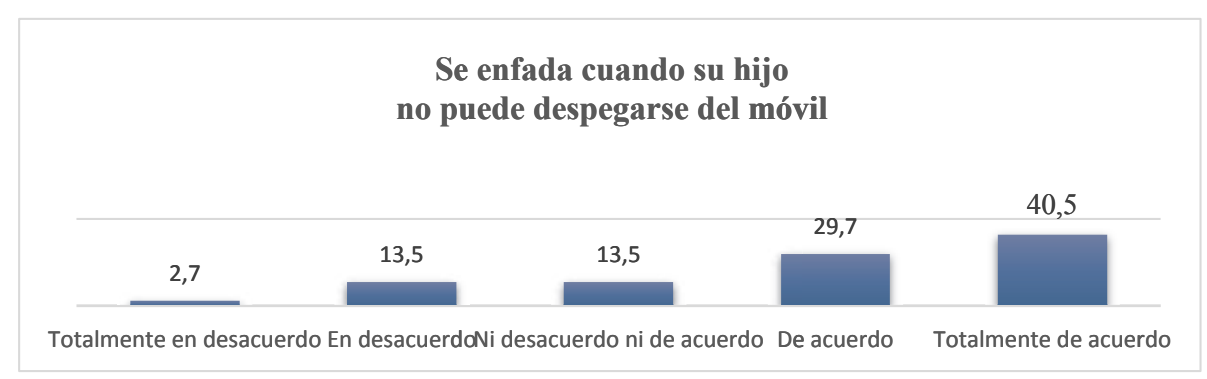

Por otra parte, el 89,1\% están "de acuerdo" o "totalmente de acuerdo" en que los padres deben asegurarse de que sus hijos utilicen el móvil de una manera apropiada (ver Figura 5). Sin embargo, llama la atención que algunos $(5,4 \%)$ consideran que no deben ser ellos quienes se aseguren de que sus hijos hacen un uso adecuado; porcentaje pequeño pero, ciertamente, preocupante. 


\section{ADOLESCENTES Y TELÉFONO MÓVIL: PERSPECTIVA DE PADRES RESIDENTES EN EL ÁMBITO RURAL}

Figura 5: Porcentajes sobre el deber de los padres

Los padres deben asegurarse de que sus hijos/as utilizan el móvil de manera adecuada

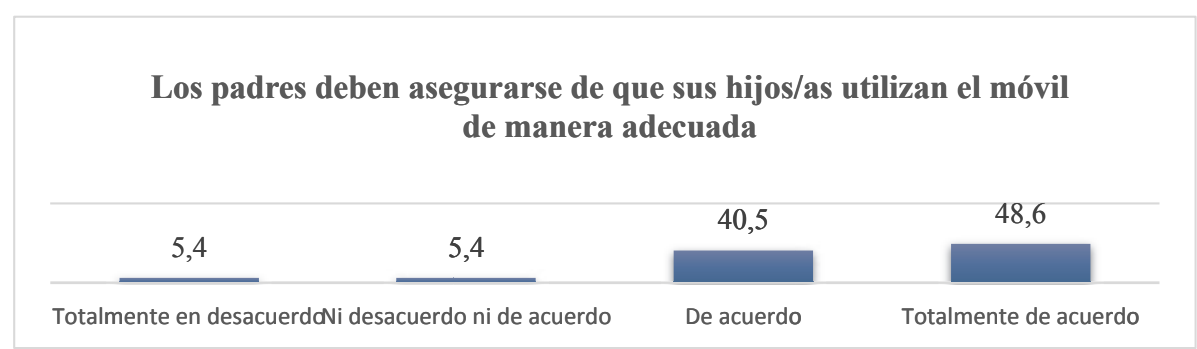

En cuanto a contar con conocimientos suficientes para ser ellos quienes eduquen en el buen uso del móvil, predominan los porcentajes situados en las respuestas negativas (Figura 6). Podemos interpretarlo como que los progenitores no se ven preparados ni capacitados para realizar, de manera apropiada, la educación sobre nuevas tecnologías, ya que refieren carecer de los conocimientos necesarios.

Figura 6: Porcentajes sobre conocimientos necesarios

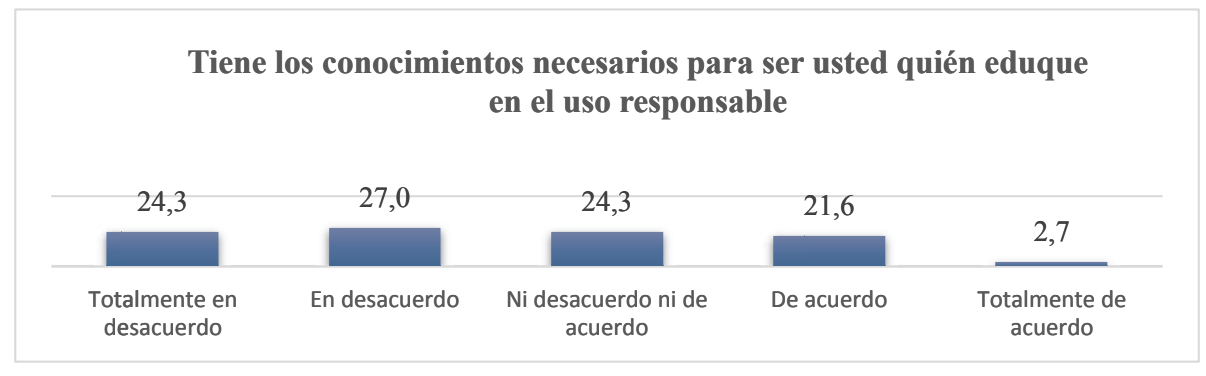

En cuanto a la opinión de los padres sobre las rutinas familiares (ver Figura 7), un 59,5\% niega que su hijo tarde en acudir a comer por usar el móvil, mientras que el $40,5 \%$ restante responde afirmativamente. Por lo que, aunque predomina el porcentaje negativo, cabe destacar que entre ambos hay poca diferencia. Por su parte, mientras un $67,6 \%$ de la muestra niega que sus descendientes utilicen el teléfono mientras comen 0 cenan, un 32,4\% lo afirma (ver Figura 8). Esto último resulta preocupante porque no se priorizan las relaciones familiares cara a cara, ya que incluso comiendo 0 cenando se utiliza el dispositivo de manera habitual. También destaca que un elevado porcentaje $51,4 \%$ de la muestra (ver Figura 9) niega que sus hijos se lleven el móvil al baño, mientras que el $48,6 \%$ restante lo afirman. Resultado curioso debido a que cuando acuden a un sitio tan íntimo como el baño, la mitad de los chavales se separa del móvil y otros tantos no lo hacen. Como se aprecia en la Figura 10, un $56,8 \%$ de la muestra opina que su hijo no prioriza las relaciones cara a cara en casa, mientras que un $43,2 \%$ afirman que sí. De esta forma, se interpreta que la mayoría de los hijos no hablan en casa con sus padres o les prestan la mínima atención, primando y anteponiendo el teléfono móvil.

Por lo tanto, atendiendo a los resultados relativos a los ítems de rutinas familiares se puede afirmar que, según la opinión de los padres, aunque el cambio no sea generalizado existe un elevado 
porcentaje de adolescentes en los que el uso del teléfono móvil ha hecho que hayan modificado sus rutinas.

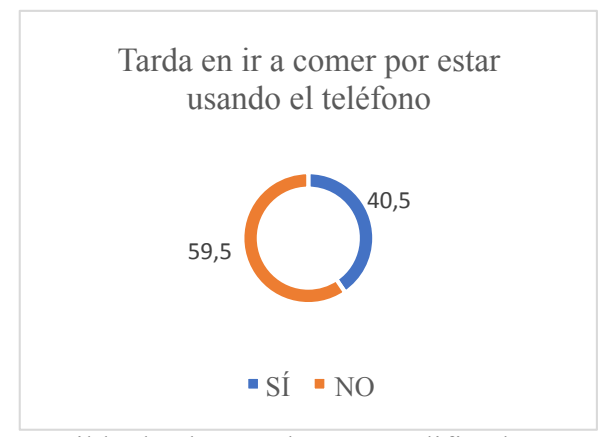

Se lo lleva al baño

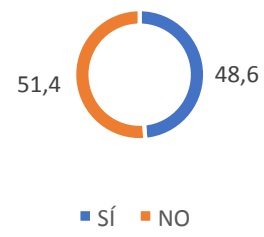

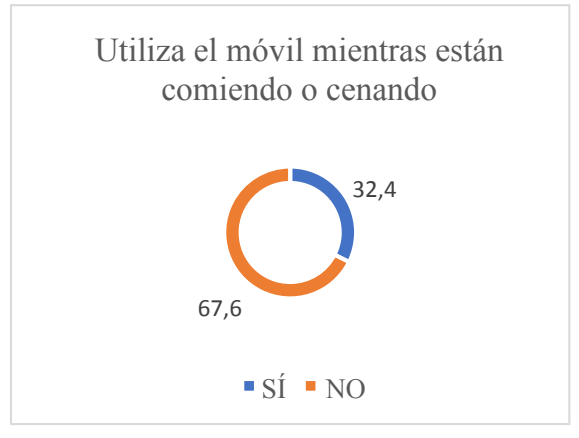

Prioriza las relaciones cara a cara en casa

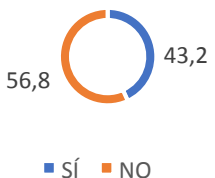

Atendiendo a la opinión parental sobre el ocio, el tiempo libre, los hobbies y los quehaceres individuales de sus hijos, un $75,7 \%$ de la muestra afirma que cuando su descendiente sale a jugar se lleva el teléfono móvil, mientras que tan sólo un $24,3 \%$ lo deja en casa (ver Figura 11). El hecho de que los chicos se lleven el teléfono con ellos puede influir en las relaciones con los demás, ya que pueden prestarle más atención al dispositivo que a los iguales, obstaculizando la relación con ellos. En la Figura 12 se aprecia cómo un $54,1 \%$ de los padres afirman que su hijo tiene delante el móvil cuando estudia o hace deberes. No afirman que lo usen, solo que lo tienen delante. Puede ser que les tranquilice tenerlo cerca, que lo utilicen para buscar información, etc. No obstante, éste es un hecho altamente significativo y a tener en cuenta, ya que puede disminuir la concentración, favorecer la dispersión, causar serias distracciones e influir negativamente en el rendimiento académico. Por su parte, cómo muestra la Figura 13 , un $54,1 \%$ de los padres afirman que lo primero que hacen sus hijos al llegar a casa del colegio es comprobar el móvil. Hecho que llama la atención si suponemos -como parece manifiesto- que lo llevan con ellos durante el día y pueden usarlo cuando quieran y, sin embargo, al llegar a casa en vez de prestar atención a sus progenitores se la dedican al teléfono. En la Figura 14 resalta que el $62,2 \%$ opina que sus hijos no dedican el mismo tiempo a las aficiones que tenían antes de poseer móvil. Hecho preocupante debido a que actividades de ocio y tiempo libre (deportivas, sociales, etc.) se pueden haber sustituido por el uso del teléfono móvil. 


\section{ADOLESCENTES Y TELÉFONO MÓVIL: PERSPECTIVA DE PADRES RESIDENTES EN EL ÁMBITO RURAL}

Tras el análisis de los ítems pertenecientes al apartado de ocio y tiempo libre individual, se puede concluir que poseer un teléfono móvil sí ha influido en el comportamiento psicosocial de los adolescentes a vista de sus padres, ya que predominan las respuestas positivas.
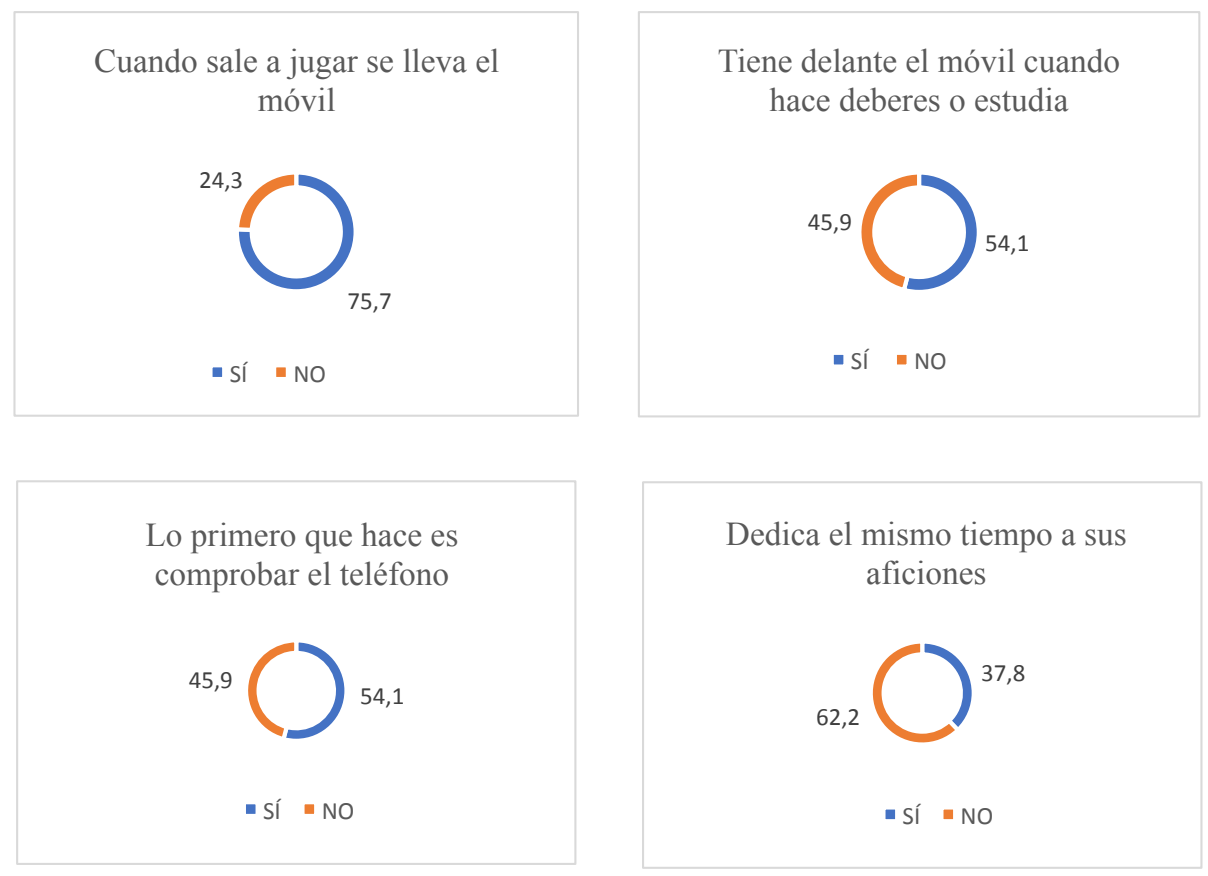

En cuanto a los ítems referidos al ocio y tiempo libre familiar, en la Figura 15 se refleja cómo un $54,1 \%$ de la muestra afirma que su descendiente está pendiente del móvil cuando ven la televisión en familia, mientras que un $45,9 \%$ lo niega. Este hecho influye en el ocio familiar, ya que no se disfruta de la misma manera el ocio compartido porque parte de la atención se la están prestando al dispositivo, priorizándolo frente al resto. También vemos como un 91,9\% (ver Figura 16) afirma que su hijo utiliza el móvil mientras realizan un trayecto en coche. Llama la atención ya que la diferencia es más que notable en este ítem: sólo un 8,1\% de los sujetos niegan que su hijo utilice el móvil cuando van en el coche. La Figura 17 muestra cómo el 51,4\% de la muestra afirma que sus hijos no dejan del teléfono, aunque no haya cobertura, sino que siguen mirándolo cada poco tiempo por si la situación hubiera cambiado y hubieran recuperado la cobertura. Mientras que el 48,6\% afirman que en tal situación se olvidan del teléfono móvil, tal vez porque ya tienen asumido que no tendrán cobertura. También vemos cómo un $51,4 \%$ (ver Figura 18) niega que su descendiente le pida el móvil para entretenerse cuando no dispone del suyo propio. Esto puede deberse a que el móvil de su progenitor no tiene las mismas aplicaciones que el suyo, por lo que no le resulta especialmente útil. Mientras que un $48,6 \%$ afirma que su hijo sí le pide el móvil cuando no dispone del suyo, evitando de esta manera socializarse. 


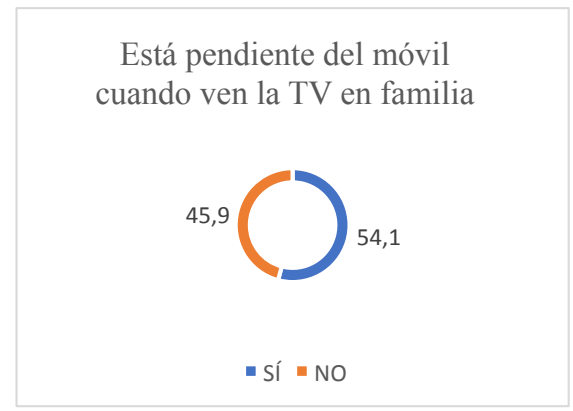

Si no hay cobertura se olvida del teléfono

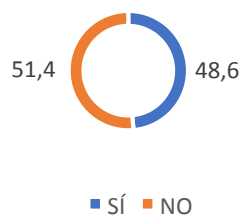

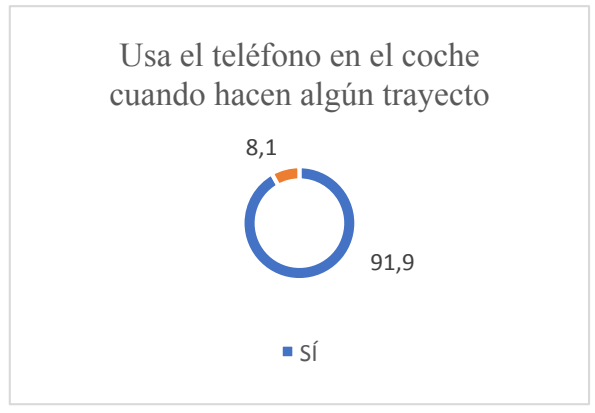

Si no tiene su móvil le pide el móvil para divertirse

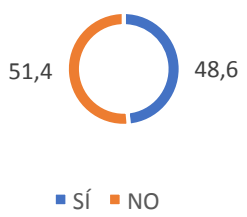

En cuanto al apartado de la existencia de reglas y limites, observamos (Figura 19) cómo tan sólo un $51,4 \%$ de la muestra afirma controlar el tiempo que su hijo usa el móvil, mientras que el $48,6 \%$ restante admite que no lo controla. Esta cierta despreocupación de los padres en este aspecto puede llegar a ser un problema, ya que los adolescentes podrían realizar un uso excesivo del dispositivo debido a la inexistencia de límites en cuanto al tiempo de uso y, además, sus padres no se enterarían de ello. También, observamos (Figura 20) cómo un 83,8\% admite haber prohibido en algún momento a su hijo el uso del teléfono móvil, mientras que el 16,2\% reconoce no haberlo hecho nunca. La inexistencia de normas y de límites al respecto puede suponer un problema, al propiciar que los menores manejen sus móviles cuando y como quieran, con escaso o nulo control parental. Respecto al ítem de si el otro miembro de la pareja pone límites en cuanto al uso del teléfono móvil (ver Figura 21), un $62,2 \%$ de la muestra afirma que su pareja no es quien pone límites, mientras que el $37,8 \%$ dice que su pareja sí pone límites y normas al respecto. Este hecho puede ser preocupante debido a que, si un miembro de la pareja no pone normas ni límites y el otro miembro tampoco, el adolescente se encuentra con la inexistencia de normas, por lo que puede hacer lo que quiera respecto al teléfono móvil, lo que pudiera derivar en un uso problemático del mismo. También destaca el hecho (ver Figura 22) de que el 54,1\% niega usar el teléfono como un premio 0 castigo para su hijo, pero el 45,9\% afirma hacerlo, cosa que puede resultar un problema ya que se da una imagen del móvil como un objeto de valor, que pueden ganar o perder dependiendo de cómo se comporten, y no como un objeto que es útil en determinadas situaciones. 


\section{ADOLESCENTES Y TELÉFONO MÓVIL: PERSPECTIVA DE PADRES RESIDENTES EN EL ÁMBITO RURAL}

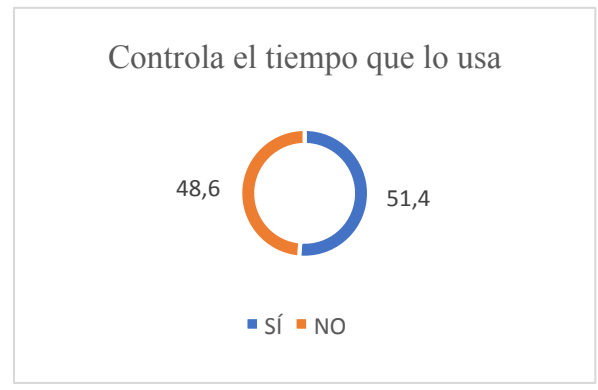

Es su pareja la que pone límites

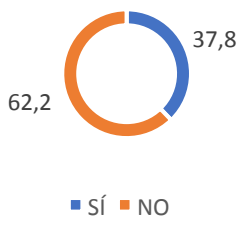

Alguna vez ha prohibido su uso

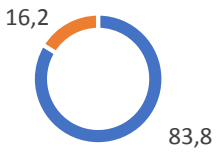

- sí - NO

Utiliza el móvil como premio o castigo

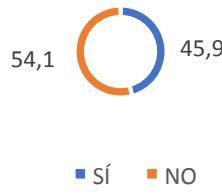

Tras el análisis de las preguntas sobre la existencia de normas y límites, se deduce que predominan los padres que no utilizan ni reglas ni límites en cuanto al uso del dispositivo móvil, lo que puede ser perjudicial ya que el menor hace el uso que quiere, cuando y cuanto quiere, sin nadie que le aconseje o le diga cuál es el uso que debería.

Por lo que respecta a los resultados sobre los ítems referidos a la existencia de formación destaca (ver Figura 23) que el $83,8 \%$ de la muestra admite no haber acudido nunca a una charla sobre educación en nuevas tecnologías, mientras que tan sólo el 16,2\% sí lo ha hecho. Esto supone un verdadero problema, ya que si los padres no tienen información y formación suficiente acerca de las nuevas tecnologías y cómo educar en ellas ¿cómo van a encontrar la manera de educar a sus hijos para que hagan un uso adecuado de las mismas? También resaltar que un $51,4 \%$ de los sujetos reconocen no haber leído ningún artículo o libro relacionado con el tema, mientras que el 48,6\% afirma haber leído algún material respecto a educar en nuevas tecnologías (ver Figura 24). Aunque entre los porcentajes no existe mucha diferencia es preocupante el hecho de que muchos padres confiesen no hacer nada por informarse sobre el tema para intentar proporcionar a sus hijos información adecuada y fiable y para que su uso de la telefonía sea positivo y no problemático. En definitiva, el $51,4 \%$ de la muestra afirma que la única información que tiene acerca de las nuevas tecnologías procede de su entorno próximo (Figura 25), hecho que es preocupante debido a que es probable que ese entorno no tenga información fiable, correcta y verídica y se originen mitos o se transmita información inadecuada. 

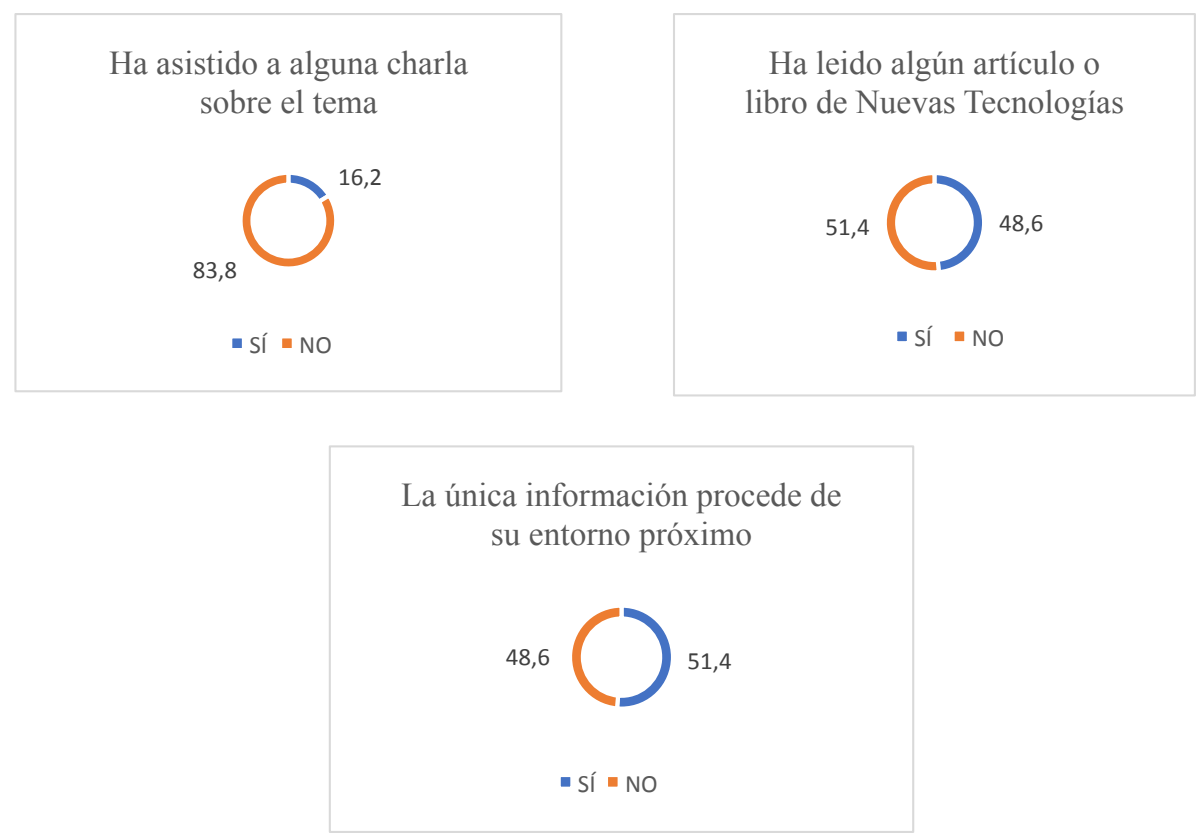

Tras el análisis de las preguntas sobre la formación parental, destaca que entre la muestra predomina una inexistencia de la misma, ya que la mayor parte sólo ha recibido información de su entorno próximo, muchos no han leído nada acerca del tema ni tampoco han acudido a ninguna charla. De tal forma que si los padres carecen de información acerca de educar en nuevas tecnologías y, entre ellas, en el uso del teléfono móvil, ¿cómo van a educar a sus hijos para hacer un uso responsable y adecuado? Por lo que se deduce que es indispensable la formación 0 , al menos, aportar a los padres pautas de actuación para que, posteriormente, eduquen a sus hijos, ya que son los que normalmente los van a acompañar durante su proceso de desarrollo.

\section{CONCLUSIONES}

Tras el análisis de los resultados podemos afirmar que, desde la perspectiva de los padres, éstos consideran mayoritariamente que el teléfono móvil ha influido en el comportamiento psicosocial de sus hijos adolescentes, provocando cambios en el mismo. En esta línea, las conclusiones que han podido extraerse a la luz del análisis de nuestra investigación son:

En primer lugar, muchos padres ven cómo las rutinas familiares que antes seguían se han visto influidas: han modificado la manera de ocupar el ocio y tiempo libre individual, la calidad del ocio y tiempo libre familiar se ha reducido y los hobbies que tenían los menores se han visto perjudicados, es decir, el teléfono móvil no sólo ha provocado cambios en sus comportamientos, sino que ha influido en su forma de vida.

En segundo lugar, se ha comprobado cómo pocos padres establecen para sus hijos reglas 0 límites de uso de los dispositivos móviles.

En tercer lugar, se ha verificado la inexistencia generalizada de formación de los padres acerca 


\section{ADOLESCENTES Y TELÉFONO MÓVIL: PERSPECTIVA DE PADRES RESIDENTES EN EL ÁMBITO RURAL}

de las nuevas tecnologías y, por lo tanto, se perciben carencias a la hora de educar a sus hijos en el tema.

En definitiva, todos aceptamos la entrada de nuevas herramientas tecnológicas en nuestras vidas sin tener en cuenta el impacto que pueden llegar a tener sobre ellas y los cambios que pueden ocasionar en nuestro entorno y en nosotros mismos.

Finalmente, cabe destacar la existencia de limitaciones en la ejecución de esta investigación, en especial la escasez muestral debida a la baja densidad de población adulta y con hijos adolescentes del ámbito rural seleccionado, aunque es de elogiar la disposición a la colaboración de todos ellos. Por este motivo sería interesante poder replicarla en otros entornos rurales para, de esta manera, poder comparar los resultados obtenidos en diferentes poblaciones.

\section{REFERENCIAS BIBLIOGRÁFICAS}

Castells, P. y de Bofarull, I. (2002). Enganchados a las pantallas: Televisión, videojuegos, internet y móviles. Guía para padres, educadores y usuarios. Barcelona: Planeta.

Cuesta, U. (2012). Uso "envolvente" del móvil en jóvenes: Propuesta de un modelo de análisis. Estudios Sobre el Mensaje Periodístico, 18, 253-262.

Echeburúa, E. y de Corral, P. (2010). Adicción a las nuevas tecnologías y a las redes sociales en jóvenes: Un nuevo reto. Adicciones, 22 (2), 91-95.

Graner, P., Beranuy, M., Sanchéz, X., Chamarro, A. y Castellana, M. (2007). ¿Qué uso hacen los jóvenes y adolescentes de Internet y del móvil? En Comunicación e Xuventude: Actas do Foro Internacional (pp. 71-90).

Lorente, S. (2002). Juventud y teléfonos móviles: Algo más que una moda. Revista de Estudios de Juventud, 57(2), 9-24.

Valdivia, C. (2008). La familia: Concepto, cambios y nuevos modelos. La Revue du REDIF, 2 (1), 1522.

Villanueva, V. (2012). Programa de prevención del abuso y la dependencia del teléfono móvil en población adolescente. (Tesis Doctoral). Universidad de Valencia, España.

Weezel, A. y Benavides, C. (2009). Uso de teléfonos móviles por los jóvenes. Cuadernos de Información, 25, 5-14. 